\title{
A MINIMAL SET OF GENERATORS FOR THE CANONICAL IDEAL OF A NON-DEGENERATE CURVE
}

\author{
WOUTER CASTRYCK and FILIP COOLS
}

(September 30, 2014)

\begin{abstract}
We give an explicit way of writing down a minimal set of generators for the canonical ideal of a non-degenerate curve, or of a more general smooth projective curve in a toric surface, in terms of its defining Laurent polynomial.
\end{abstract}

Accompanying Magma file: canonical.m

\section{Introduction}

Let $k$ be an algebraically closed field and consider the affine torus $\mathbb{T}^{2}=$ $(k \backslash\{0\})^{2}$. Let $\Delta \subset \mathbb{R}^{2}$ be a two-dimensional lattice polygon and define $N=\sharp\left(\Delta \cap \mathbb{Z}^{2}\right)$. In this article we are concerned with algebraic curves $U_{f} \subset \mathbb{T}^{2}$ that are cut out by a sufficiently generic Laurent polynomial

$$
f=\sum_{(i, j) \in \Delta \cap \mathbb{Z}^{2}} c_{i, j} x^{i} y^{j} \in k\left[x^{ \pm 1}, y^{ \pm 1}\right] .
$$

Here 'sufficiently generic' means that $f$ is contained in a certain Zariski dense subset of the corresponding $N$-dimensional coefficient space. More precisely, to each $(i, j) \in \Delta \cap \mathbb{Z}^{2}$ we associate a formal variable $X_{i, j}$, and we let

$$
\mathbb{P}^{N-1}=\operatorname{Proj} k\left[X_{i, j}\right]_{(i, j) \in \Delta \cap \mathbb{Z}^{2}} .
$$

We have a natural embedding

$$
\varphi_{\Delta}: \mathbb{T}^{2} \hookrightarrow \mathbb{P}^{N-1}:(x, y) \mapsto\left(x^{i} y^{j}\right)_{(i, j) \in \Delta \cap \mathbb{Z}^{2}},
$$

(c) XXXX Australian Mathematical Society $0263-6115 / \mathrm{XX} \$ \mathrm{~A} 2.00+0.00$ 
the Zariski closure of the image of which is a toric surface that we denote by $\operatorname{Tor}(\Delta)$. Note that $\varphi_{\Delta}\left(U_{f}\right)$ is contained in the hyperplane section

$$
H: \sum_{(i, j) \in \Delta \cap \mathbb{Z}^{2}} c_{i, j} X_{i, j}=0
$$

of $\operatorname{Tor}(\Delta) \subset \mathbb{P}^{N-1}$. Then by 'sufficiently generic' we mean that the Zariski closure $C_{f}$ of $\varphi_{\Delta}\left(U_{f}\right)$ is a smooth projective curve that equals this hyperplane section. Bertini's theorem implies that this is indeed a Zariski dense condition. Alternatively and more explicitly, for $C_{f}$ to arise as a smooth hyperplane section of $\operatorname{Tor}(\Delta)$, it suffices that $f$ is non-degenerate with respect to $\Delta$, in the sense that for each face $\tau \subset \Delta$ (vertex, edge, or $\Delta$ itself) the system

$$
f_{\tau}=\frac{\partial f_{\tau}}{\partial x}=\frac{\partial f_{\tau}}{\partial y}=0
$$

has no solutions in $\mathbb{T}^{2}$. Here $f_{\tau}$ is obtained from $f$ by restricting to those terms that are supported on $\tau$. Non-degeneracy is known to be generically satisfied; see [4, Prop. 1].

REMARK. Every (nef and big) smooth projective curve $C$ on a toric surface $X$ arises as such a toric hyperplane section. Indeed, let $D_{C}$ be a torus-invariant divisor on $X$ that is linearly equivalent to $C$, and let $\Delta$ be the two-dimensional lattice polygon associated to $D_{C}$ (here we use that $C$ is nef and big). Then the $\mathbb{T}^{2}$-part of $C$ is cut out by a Laurent polynomial $f \in k\left[x^{ \pm 1}, y^{ \pm 1}\right]$ that is supported on $\Delta$. The above construction then yields a hyperplane section $C_{f}$ of $\operatorname{Tor}(\Delta)$ that is isomorphic to $C$.

We refer to $[3, \S 3-4]$ and the references therein for more background, both on curves in toric surfaces and on non-degenerate Laurent polynomials. Various of these references assume the base field $k$ to be of characteristic 0 , but we emphasize that the material presented below is valid in any characteristic.

The main result of this paper is an explicit recipe for writing down a minimal set of generators for the canonical ideal of curves of the form $C_{f}$, where $f \in k\left[x^{ \pm 1}, y^{ \pm 1}\right]$ satisfies the above generic condition (e.g. nondegeneracy) with respect to a given two-dimensional lattice polygon $\Delta$.

A quick implementation of the resulting algorithm already heavily outperforms Magma's built-in function for computing canonical ideals [1]. The latter relies on general lattice basis reduction algorithms that were developed by Hess [9]. Our code can be found in the file canonical.m that accompanies the article. It allows one to compute the canonical ideal of a non-degenerate curve of genus $g \approx 100$ in a matter of minutes, whereas everything beyond $g=20$ looks hopeless using the Magma intrinsic, both in terms of time and memory. Of course, this comes at the cost of working in 
less generality, but note that the condition of non-degeneracy is generically satisfied (for a fixed instance of $\Delta$ ), and easy to verify in our range of interest. It therefore seems useful to begin the computation of the canonical ideal with a test for whether the input polynomial is non-degenerate or not, and if yes, to proceed with the method presented here.

Our starting point is a theorem by Hovanskir [10], stating that there exists a canonical divisor $K_{\Delta}$ on $C_{f}$ such that a basis for $H^{0}\left(C_{f}, K_{\Delta}\right)$ is given by

$$
\left\{x^{i} y^{j}\right\}_{(i, j) \in \Delta^{(1)} \cap \mathbb{Z}^{2}},
$$

where $\Delta^{(1)}$ denotes the convex hull of the interior lattice points of $\Delta$. Here $x, y$ are viewed as functions on $C_{f}$ through $\varphi_{\Delta}$. See [5, Prop. 10.5.8] for a modern proof. Two notable corollaries are:

- The genus of $C_{f}$ equals $g=\sharp\left(\Delta^{(1)} \cap \mathbb{Z}^{2}\right)$.

- If $g \geq 2$ then the linear system $\left|K_{\Delta}\right|$ maps $U_{f}$ inside the image of $\varphi_{\Delta^{(1)}}$. In particular:

- if $\Delta^{(1)}$ is one-dimensional then the canonical image of $C_{f}$ is a rational normal curve of degree $g-1$, hence $C_{f}$ is hyperelliptic;

- if $\Delta^{(1)}$ is two-dimensional, then $C_{f}$ is non-hyperelliptic and the canonical image of $C_{f}$ is contained in the toric surface $\operatorname{Tor}\left(\Delta^{(1)}\right) \subset$ $\mathbb{P}^{g-1}$.

See $[3, \S 4]$ and its references for more details.

In what follows we assume that $C_{f}$ is non-hyperelliptic or, equivalently, that $\Delta^{(1)}$ is two-dimensional. Then the generators for the canonical ideal of $C_{f}$ are gathered in two steps.

- In Section 2, which can be seen as an addendum to previous work by Koelman [13, 12], we will describe a method for finding a minimal set of generators for the ideal of $\operatorname{Tor}\left(\Delta^{(1)}\right)$. We also provide explicit formulas for the number of generators in each degree. Because of the independent interest, we will do this for toric surfaces $\operatorname{Tor}(\Gamma)$ where $\Gamma$ is an arbitrary two-dimensional lattice polygon (not necessarily of the form $\left.\Delta^{(1)}\right)$.

- Then in Section 3, we will explicitly describe which generators have to be added in order to obtain a minimal set of generators for the canonical ideal of $C_{f}$. These can be seen as analogues of Reid's rolling factors [15], where the 'rolling' now happens in two directions, rather than one.

Notation AND TERMinology. We use a special notation for two recurring polygons

$$
\Sigma=\operatorname{conv}\{(0,0),(1,0),(0,1)\}, \quad \Upsilon=\operatorname{conv}\{(-1,-1),(1,0),(0,1)\},
$$


and write $\cong$ to indicate unimodular equivalence. For instance, $\Delta \cong \Sigma$ if and only if $\Delta$ is a unimodular simplex. We recall that the convex hull of the interior lattice points of a two-dimensional lattice polygon $\Delta$ is denoted by $\Delta^{(1)}$. If the latter is again two-dimensional, we abbreviate $\Delta^{(1)(1)}$ by $\Delta^{(2)}$. We use $\Delta^{\circ}$ to denote the topological interior of $\Delta$, and write $\partial \Delta$ for its boundary. A two-dimensional lattice polygon $\Delta$ is said to be hyperelliptic if $\Delta^{(1)}$ is one-dimensional. If $X$ is a projectively embedded variety over $k$, we write $\mathcal{I}(X)$ for its defining ideal. For each non-negative integer $d$ we use $\mathcal{I}_{d}(X)$ to denote the $k$-vector space of homogeneous degree $d$ polynomials that are contained in $\mathcal{I}(X)$.

ACKnowledgement. We would like to thank the anonymous referee for some helpful remarks.

\section{The ideal of a toric surface}

Let $\Gamma \subset \mathbb{R}^{2}$ be a two-dimensional lattice polygon and let $N=\#\left(\Gamma \cap \mathbb{Z}^{2}\right)$. Define $\operatorname{Tor}(\Gamma)$ as the Zariski closure inside $\mathbb{P}^{N-1}$ of the image of $\varphi_{\Gamma}$. A result due to Koelman $[12,13]$ states that the ideal $\mathcal{I}(\operatorname{Tor}(\Gamma))$ is generated by all binomials

$$
\prod_{\ell=1}^{n} X_{i_{\ell}, j_{\ell}}-\prod_{\ell=1}^{n} X_{i_{\ell}^{\prime}, j_{\ell}^{\prime}} \quad \text { for which } \quad \sum_{\ell=1}^{n}\left(i_{\ell}, j_{\ell}\right)=\sum_{\ell=1}^{n}\left(i_{\ell}^{\prime}, j_{\ell}^{\prime}\right)
$$

where $n \in\{2,3\}$. Moreover one can restrict to $n=2$ if and only if $\sharp\left(\partial \Gamma \cap \mathbb{Z}^{2}\right) \geq 4$ or $\Gamma$ is a unimodular simplex. This result was generalized to property $N_{p}$ for arbitrary $p$ by Hering and Schenck; see [8, Thm.4.20].

The current section can be seen as an addendum to Koelman's work: we give explicit formulas for the number of quadrics and cubics in a minimal set of homogeneous generators for $\mathcal{I}(\operatorname{Tor}(\Gamma))$.

Lemma 2.1. For all integers $d \geq 0$ one has:

$$
\operatorname{dim} \mathcal{I}_{d}(\operatorname{Tor}(\Gamma))=\left(\begin{array}{c}
\sharp\left(\Gamma \cap \mathbb{Z}^{2}\right)+d-1 \\
d
\end{array}\right)-\sharp\left(d \Gamma \cap \mathbb{Z}^{2}\right) .
$$

Proof. The $k$-vector space morphism

$$
\chi_{d}: \mathcal{I}_{d}\left(\mathbb{P}^{N-1}\right) \rightarrow k\left[x^{ \pm 1}, y^{ \pm 1}\right]: X_{i_{1}, j_{1}} \cdots X_{i_{d}, j_{d}} \mapsto x^{i_{1}+\cdots+i_{d}} y^{j_{1}+\cdots+j_{d}}
$$

has kernel $\mathcal{I}_{d}(\operatorname{Tor}(\Gamma))$ and surjects onto $\left\langle x^{i} y^{j}\right\rangle_{(i, j) \in d \Gamma \cap \mathbb{Z}^{2}}$ (here we use that two-dimensional lattice polygons are always normal [2, Prop. 1.2.2-4], i.e. every lattice point in $d \Gamma$ is the sum of $d$ lattice points in $\Gamma$ ).

The main result of this section is: 
Theorem 2.2. A minimal set of generators for $\mathcal{I}(\operatorname{Tor}(\Gamma))$ consists of

$$
\left(\begin{array}{c}
\sharp\left(\Gamma \cap \mathbb{Z}^{2}\right)+1 \\
2
\end{array}\right)-\sharp\left(2 \Gamma \cap \mathbb{Z}^{2}\right) \text { quadrics and } c_{\Gamma} \text { cubics, }
$$

where

$$
c_{\Gamma}= \begin{cases}0 & \text { if } \sharp\left(\partial \Gamma \cap \mathbb{Z}^{2}\right) \geq 4 \text { or } \Gamma \cong \Sigma, \\ 1 & \text { if } \sharp\left(\partial \Gamma \cap \mathbb{Z}^{2}\right)=3, \Gamma \neq \Sigma, \Gamma \text { is non-hyperelliptic, } \\ \sharp\left(\Gamma \cap \mathbb{Z}^{2}\right)-3 & \text { if } \sharp\left(\partial \Gamma \cap \mathbb{Z}^{2}\right)=3, \Gamma \neq \Sigma, \Gamma \text { is hyperelliptic. }\end{cases}
$$

Proof. The formula for the number of quadrics follows from Lemma 2.1 along with the fact that $\operatorname{Tor}(\Gamma)$ is not contained in any hyperplane of $\mathbb{P}^{N-1}$. By Koelman's result, it remains to prove the formula for the number of cubics $c_{\Gamma}$ when $\sharp\left(\partial \Gamma \cap \mathbb{Z}^{2}\right)=3$ and $\Gamma \not \Sigma$. We moreover know that $c_{\Gamma} \geq 1$ in these cases. Also recall that $\mathcal{I}(\operatorname{Tor}(\Gamma))$ is generated by binomials.

First assume that $\Gamma$ is non-hyperelliptic and $\Gamma \neq \Upsilon$. Along with $\sharp(\partial \Gamma \cap$ $\left.\mathbb{Z}^{2}\right)=3$ and $\Gamma \neq \Sigma$ this implies that $\Gamma^{(1)}$ is two-dimensional; see e.g. Koelman's classification [11, Ch.4], although this could also serve as an easy exercise. Let $\left\{v_{1}, v_{2}, v_{3}\right\}$ be the three vertices of $\Gamma$ and consider

$$
\Gamma^{\prime}=\operatorname{conv}\left(\left(\Delta \backslash\left\{v_{1}\right\}\right) \cap \mathbb{Z}^{2}\right) .
$$

Then $\Gamma^{\prime} \supset \Gamma^{(1)}$ is again a two-dimensional lattice polygon. We claim that there are at least 4 lattice points on its boundary. Indeed, if there would only be 3 such lattice points, then $\Gamma^{\prime}$ would be a triangle whose vertices are $\left\{v, v_{2}, v_{3}\right\}$, where $v$ is contained in the interior of $\Gamma$, and the triangles $v_{1}-v-v_{2}$ and $v_{1}-v-v_{3}$ are unimodular simplices (i.e. they do not contain any lattice points besides the vertices).

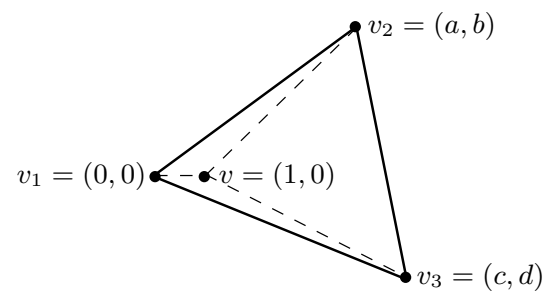

We may assume that $v_{1}=(0,0), v=(1,0), v_{2}=(a, b)$ and $v_{3}=(c, d)$, where $b>0>d$. By Pick's theorem the unimodularity of $v_{1}-v-v_{2}$ and $v_{1}-v-v_{3}$ implies that $b=1$ and $d=-1$, and hence that $\Gamma$ is contained in a horizontal strip of width 2: a contradiction with the fact that $\Gamma^{(1)}$ is two-dimensional. So the claim follows. Now consider a binomial

$$
C=X_{i_{1}, j_{1}} X_{i_{2}, j_{2}} X_{i_{3}, j_{3}}-X_{i_{1}^{\prime}, j_{1}^{\prime}} X_{i_{2}^{\prime}, j_{2}^{\prime}} X_{i_{3}^{\prime}, j_{3}^{\prime}} \in \mathcal{I}_{3}(\operatorname{Tor}(\Gamma))
$$

and define $\Gamma_{C}=\operatorname{conv}\left\{\left(i_{1}, j_{1}\right),\left(i_{2}, j_{2}\right),\left(i_{3}, j_{3}\right),\left(i_{1}^{\prime}, j_{1}^{\prime}\right),\left(i_{2}^{\prime}, j_{2}^{\prime}\right),\left(i_{3}^{\prime}, j_{3}^{\prime}\right)\right\}$. 
- If $\Gamma_{C} \subsetneq \Gamma$, then by the above $\Gamma_{C} \subset \Gamma^{\prime}$ for a subpolygon $\Gamma^{\prime}$ that contains at least 4 lattice points on the boundary. So by Koelman's result applied to $\Gamma^{\prime}$ our cubic $C$ can be written as a linear combination of a number of elements of $\mathcal{I}_{2}(\operatorname{Tor}(\Gamma))$.

- If $\Gamma_{C}=\Gamma$ then it is not hard to see that either $\left(i_{1}, j_{1}\right),\left(i_{2}, j_{2}\right),\left(i_{3}, j_{3}\right)$ or $\left(i_{1}^{\prime}, j_{1}^{\prime}\right),\left(i_{2}^{\prime}, j_{2}^{\prime}\right),\left(i_{3}^{\prime}, j_{3}^{\prime}\right)$ are the three vertices of $\Gamma$; see [12, Lem. 2.6].

It follows that either the sum or difference of two binomials $C_{1}, C_{2} \in \mathcal{I}_{3}(\operatorname{Tor}(\Gamma))$ that are independent of $\mathcal{I}_{2}(\operatorname{Tor}(\Gamma))$ is again a cubic binomial $C$. But the latter satisfies $\Gamma_{C} \subsetneq \Gamma$, so by the first observation $C$ is expressible as a linear combination of elements of $\mathcal{I}_{2}(\operatorname{Tor}(\Gamma))$. This proves that one cubic is sufficient, i.e. $c_{\Gamma}=1$.

Next assume that $\Gamma$ is hyperelliptic or $\Gamma \cong \Upsilon$. Using that $\sharp\left(\partial \Gamma \cap \mathbb{Z}^{2}\right)=3$ we find that it is unimodularly equivalent to

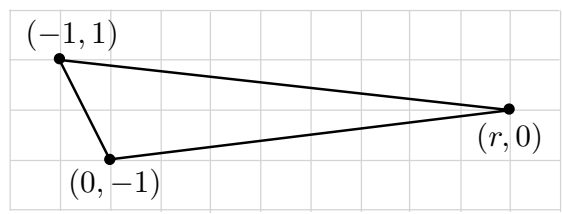

where $r=\#\left(\Gamma \cap \mathbb{Z}^{2}\right)-3$. One verifies that the irreducible binomials in $\mathcal{I}_{3}(\operatorname{Tor}(\Gamma))$ involving $X_{-1,1}$ or $X_{0,-1}$ must involve both variables in the same monomial. This monomial is necessarily among

$$
X_{-1,1} X_{0,-1} X_{i, 0} \quad i=1, \ldots, r
$$

and conversely, for each of these monomials one may choose a corresponding binomial $C_{i} \in \mathcal{I}_{3}(\operatorname{Tor}(\Gamma))$. As before we find that the difference or sum of two cubic binomials involving the same monomial $X_{-1,1} X_{0,-1} X_{i, 0}$ is a linear combination of elements of $\mathcal{I}_{2}(\operatorname{Tor}(\Gamma))$. So we conclude that $\mathcal{I}(\operatorname{Tor}(\Gamma))$ is generated by $\mathcal{I}_{2}(\operatorname{Tor}(\Gamma)) \cup\left\{C_{1}, \ldots, C_{r}\right\}$. Because the quadratic binomials in $\mathcal{I}(\operatorname{Tor}(\Gamma))$ do neither involve $X_{-1,1}$ nor $X_{0,-1}$, the latter $r$ cubics are independent of $\mathcal{I}_{2}(\operatorname{Tor}(\Gamma))$.

We have included Magma code for computing such a minimal set of (binomial) generators; see our accompanying file canonical.m. As for the quadratic generators, this is done by naively gathering all relations of the form

$$
\left(i_{1}, j_{1}\right)+\left(i_{2}, j_{2}\right)=\left(i_{1}^{\prime}, j_{1}^{\prime}\right)+\left(i_{2}^{\prime}, j_{2}^{\prime}\right)
$$

for $\left(i_{1}, j_{1}\right),\left(i_{2}, j_{2}\right),\left(i_{1}^{\prime}, j_{1}^{\prime}\right),\left(i_{2}^{\prime}, j_{2}^{\prime}\right) \in \Gamma \cap \mathbb{Z}^{2}$, and then finding a $k$-linearly independent subset of the set of corresponding binomials

$$
X_{i_{1}, j_{1}} X_{i_{2}, j_{2}}-X_{i_{1}^{\prime}, j_{1}^{\prime}} X_{i_{2}^{\prime}, j_{2}^{\prime}}
$$


In the case where $\sharp\left(\partial \Gamma \cap \mathbb{Z}^{2}\right)=3, \Gamma \neq \Sigma$ and $\Gamma$ is non-hyperelliptic, a single binomial of the form $(1)$ with $\left(i_{1}, j_{1}\right),\left(i_{2}, j_{2}\right),\left(i_{3}, j_{3}\right)$ the vertices of $\Gamma$ is added by exhaustive search. In the hyperelliptic case the explicit construction from the above proof is followed.

EXAMPLE. The code below carries this out for the following lattice polygon $($ over $k=\overline{\mathbb{Q}})$ :
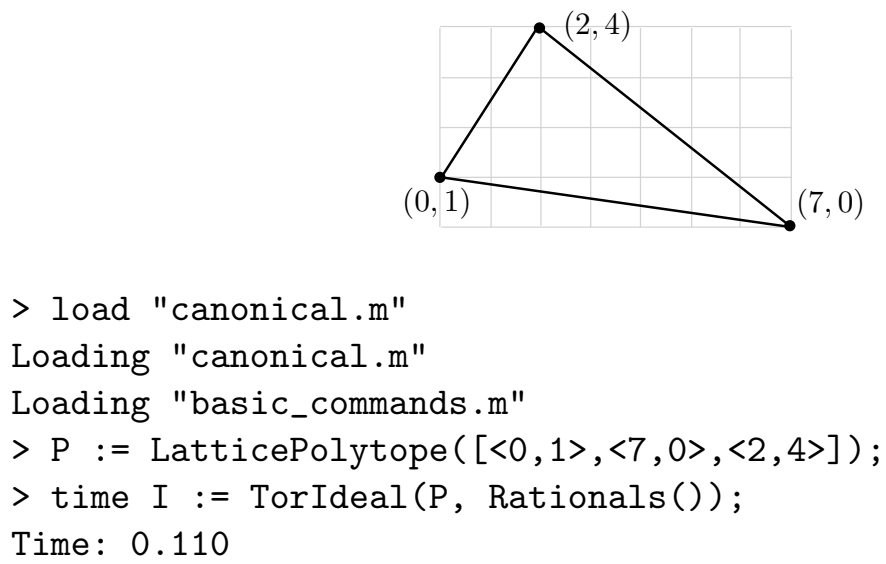

This can be used as input to more advanced functions, such as the Magma intrinsic for computing the Betti diagram:

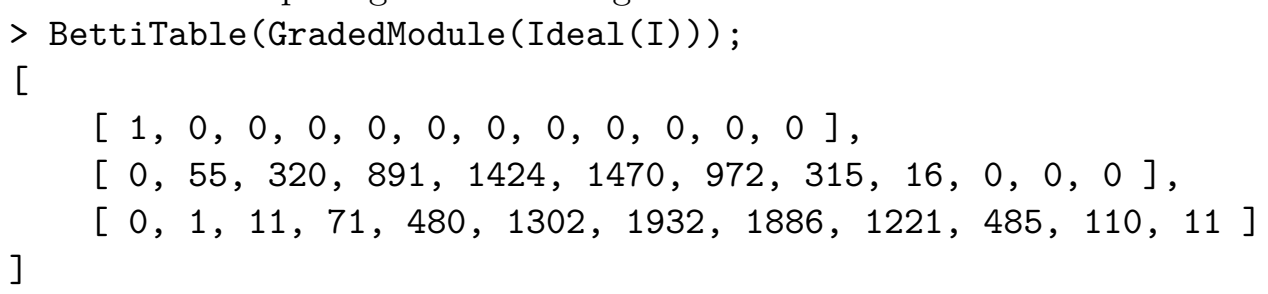

Remark. From the point of view of efficiency the above method leaves room for improvement. Especially the gathering of the quadratic generators can be done more systematically, for instance using Gröbner bases computations. These are implicitly invoked by the code below (a continuation of the above example):

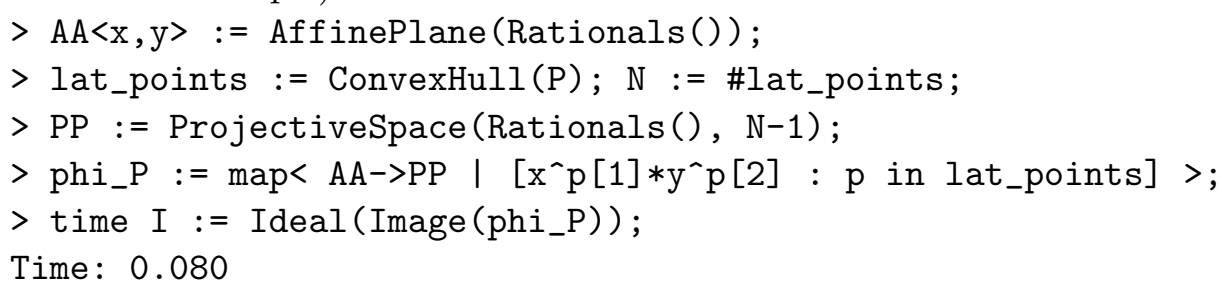

This produces a reduced Gröbner basis for Tor $(\Gamma)$. In general this is not a minimal set of generators, but its quadratic elements do form a basis of $\mathcal{I}_{2}(\operatorname{Tor}(\Gamma))$, so that one can obtain a minimal set of generators by proceeding as above. 
REMARK. Up to unimodular equivalence, the only two-dimensional instances of $\Delta^{(1)}$ for which $\sharp\left(\partial \Delta^{(1)} \cap \mathbb{Z}^{2}\right)=3$ are $\Sigma$ and $\Upsilon$. This can be shown using [7, Lem.9-11]. Therefore, for the purposes of describing the canonical ideal of curves in toric surfaces, the above general treatment is more elaborate than needed. We have included it because we believe it to be of independent interest.

\section{An explicit description of the canonical ideal}

Let $\Delta$ be a two-dimensional lattice polygon and let $f \in k\left[x^{ \pm 1}, y^{ \pm 1}\right]$ be a Laurent polynomial satisfying the sufficiently generic condition from the introduction (e.g. non-degeneracy). Assume that the corresponding curve $C_{f}$ is non-hyperelliptic of genus $g \geq 3$, i.e. $\Delta^{(1)}$ is two-dimensional and $\sharp\left(\partial \Delta^{(1)} \cap \mathbb{Z}^{2}\right) \geq 3$. Let $C_{f}^{\text {can }}$ be the canonical model of $C_{f}$ obtained using $\left|K_{\Delta}\right|$.

We already know that $\mathcal{I}\left(C_{f}^{\text {can }}\right)$ contains $\mathcal{I}\left(\operatorname{Tor}\left(\Delta^{(1)}\right)\right)$, and from the previous section we know how to find a minimal set of generators for the latter. In this section we describe which generators have to be added in order to obtain a minimal set of generators for $\mathcal{I}\left(C_{f}^{\text {can }}\right)$. A priori, it is not entirely trivial that it suffices to merely add some generators, but note from the previous remark that $\operatorname{Tor}\left(\Delta^{(1)}\right)$ is almost always generated by quadrics, in which case this is clear. The only exception is when $\Delta^{(1)} \cong \Upsilon$, which corresponds to curves of genus 4 , and is therefore well-understood.

Our main auxiliary tool is:

THEOREm 3.1. The equality

$$
\operatorname{dim} \mathcal{I}_{d}\left(C_{f}^{\text {can }}\right)-\operatorname{dim} \mathcal{I}_{d}\left(\operatorname{Tor}\left(\Delta^{(1)}\right)\right)=\sharp\left(\left((d-1) \Delta^{(1)}\right)^{(1)} \cap \mathbb{Z}^{2}\right)
$$

holds for all integers $d \geq 2$.

Proof. From Lemma 2.1 it follows that

$$
\operatorname{dim} \mathcal{I}_{d}\left(\operatorname{Tor}\left(\Delta^{(1)}\right)\right)=\left(\begin{array}{c}
g+d-1 \\
d
\end{array}\right)-\sharp\left(d \Delta^{(1)} \cap \mathbb{Z}^{2}\right) .
$$

On the other hand, let $H(d)$ be the Hilbert function of the homogeneous coordinate ring of $C_{f}^{\mathrm{can}} \subset \mathbb{P}^{g-1}$. Then $H(d)=(2 g-2) d+(1-g)=$ $(2 d-1)(g-1)$ if $d \geq 2$ (see [6, Cor. 9.4]), hence

$$
\operatorname{dim} \mathcal{I}_{d}(C)=\left(\begin{array}{c}
g+d-1 \\
d
\end{array}\right)-(2 d-1)(g-1) .
$$

So we are left with proving that

$$
\sharp\left(d \Delta^{(1)} \cap \mathbb{Z}^{2}\right)-\sharp\left(\left((d-1) \Delta^{(1)}\right)^{(1)} \cap \mathbb{Z}^{2}\right)=(2 d-1)(g-1) .
$$


For this, write $R^{(1)}=\sharp\left(\partial \Delta^{(1)} \cap \mathbb{Z}^{2}\right)$ and consider the Ehrhart polynomial

$$
\operatorname{Ehr}_{\Delta^{(1)}}(k)=\operatorname{Vol}\left(\Delta^{(1)}\right) \cdot k^{2}+\frac{R^{(1)}}{2} \cdot k+1
$$

of $\Delta^{(1)}$; see $[5, \S 9.4]$. Since $\sharp\left(k \Delta^{(1)} \cap \mathbb{Z}^{2}\right)=\operatorname{Ehr}_{\Delta^{(1)}}(k)$ and $\sharp\left(\partial\left(k \Delta^{(1)}\right) \cap \mathbb{Z}^{2}\right)=$ $k R^{(1)}$ for all $k \in \mathbb{Z}_{\geq 1}$, we have that

$$
\begin{aligned}
& \sharp\left(d \Delta^{(1)} \cap \mathbb{Z}^{2}\right)-\sharp\left(\left((d-1) \Delta^{(1)}\right)^{(1)} \cap \mathbb{Z}^{2}\right) \\
& =\operatorname{Ehr}_{\Delta^{(1)}}(d)-\operatorname{Ehr}_{\Delta^{(1)}}(d-1)+\sharp\left(\partial\left((d-1) \Delta^{(1)}\right) \cap \mathbb{Z}^{2}\right) \\
& =(2 d-1)\left(\operatorname{Vol}\left(\Delta^{(1)}\right)+\frac{R^{(1)}}{2}\right) \\
& =(2 d-1)(g-1) .
\end{aligned}
$$

This concludes the proof.

REMARK. Some readers may prefer the following cohomological proof of Theorem 3.1 (brief). Assume for ease of exposition that $\operatorname{Tor}(\Delta)$ is smooth; if not the argument below has to be preceded by a toric blow-up. Let $D_{C_{f}}$ be a torus-invariant divisor on $\operatorname{Tor}(\Delta)$ that is linearly equivalent to $C_{f}$, let $K$ be a torus-invariant canonical divisor on $\operatorname{Tor}(\Delta)$, and define $L=D_{C_{f}}+K$. When tensoring the exact sequence

$$
0 \rightarrow \mathcal{O}_{\operatorname{Tor}(\Delta)}\left(-D_{C_{f}}\right) \rightarrow \mathcal{O}_{\operatorname{Tor}(\Delta)} \rightarrow \mathcal{O}_{C_{f}} \rightarrow 0
$$

with $\mathcal{O}_{\operatorname{Tor}(\Delta)}(d L)$, taking cohomology and using the standard toric vanishing theorems for $H^{1}$ we get

$$
0 \rightarrow H^{0}(\operatorname{Tor}(\Delta),(d-1) L+K) \rightarrow H^{0}(\operatorname{Tor}(\Delta), d L) \rightarrow H^{0}\left(C_{f},\left.d L\right|_{C_{f}}\right) \rightarrow 0 .
$$

The respective dimensions of these spaces are seen to be

$$
\sharp\left(\left((d-1) \Delta^{(1)}\right)^{(1)} \cap \mathbb{Z}^{2}\right), \operatorname{dim} \frac{\mathcal{I}_{d}\left(\mathbb{P}^{g-1}\right)}{\mathcal{I}_{d}\left(\operatorname{Tor}\left(\Delta^{(1)}\right)\right)}, \text { and } \operatorname{dim} \frac{\mathcal{I}_{d}\left(\mathbb{P}^{g-1}\right)}{\mathcal{I}_{d}\left(C_{f}^{\text {can }}\right)},
$$

(indeed, by adjunction theory $\left.L\right|_{C_{f}}$ is a canonical divisor on $C_{f}$ ), so that the theorem follows.

Write

$$
f=\sum_{(i, j) \in \Delta \cap \mathbb{Z}^{2}} c_{i, j} x^{i} y^{j} \in k\left[x^{ \pm 1}, y^{ \pm 1}\right]
$$

and define $\mathcal{W}_{d}=\left(\Delta^{(1)}\right)^{\circ} \cap\left(\frac{1}{d-1} \mathbb{Z}\right)^{2}$. Note that

$$
\sharp \mathcal{W}_{d}=\sharp\left(\left((d-1) \Delta^{(1)}\right)^{(1)} \cap \mathbb{Z}^{2}\right) .
$$


To every $w \in \mathcal{W}_{d}$ we can associate a homogeneous degree $d$ polynomial, as follows. For each $(i, j) \in \Delta \cap \mathbb{Z}^{2}$ there exist

$$
v_{1,(i, j)}, \ldots, v_{d,(i, j)} \in \Delta^{(1)} \cap \mathbb{Z}^{2}
$$

such that

$$
(i, j)-w=\left(v_{1,(i, j)}-w\right)+\ldots+\left(v_{d,(i, j)}-w\right) .
$$

This follows from the inclusion $\left((d-1) \Delta^{(1)}\right)^{(1)}+\Delta \subset d \Delta^{(1)}$ and the normality of the polygon $\Delta^{(1)}$. The $d$-form

$$
\mathcal{F}_{d, w}=\sum_{(i, j) \in \Delta \cap \mathbb{Z}^{2}} c_{i, j} X_{v_{1,(i, j)}} \cdots X_{v_{d,(i, j)}}
$$

is well-defined modulo the ideal of $\operatorname{Tor}\left(\Delta^{(1)}\right)$. It clearly vanishes on $\varphi_{\Delta^{(1)}}\left(U_{f}\right)$, hence it is contained in the ideal of $C_{f}^{\text {can }}$.

The forms $\mathcal{F}_{d, w}$ with $w \in \mathcal{W}_{d}$ are $k$-linearly independent of each other and of the forms in $\mathcal{I}_{d}\left(\operatorname{Tor}\left(\Delta^{(1)}\right)\right)$. Indeed, this holds because

$$
\chi_{d}\left(\mathcal{F}_{d, w}\right)=(x, y)^{(d-1) w} \cdot f,
$$

where $\chi_{d}$ is the vector space morphism from the proof of Lemma 2.1; here we used multi-index notation, i.e. $(x, y)^{(a, b)}$ should be read as $x^{a} y^{b}$. Hence any linear combination in which the $\mathcal{F}_{d, w}$ 's appear non-trivially is mapped to a non-zero multiple of $f$, and must therefore be non-zero itself. By Theorem 3.1 , we can conclude that a basis for $\mathcal{I}_{d}\left(C_{f}^{\text {can }}\right)$ is obtained by adjoining $\left\{\mathcal{F}_{d, w}\right\}_{w \in \mathcal{W}_{d}}$ to a basis for $\mathcal{I}_{d}\left(\operatorname{Tor}\left(\Delta^{(1)}\right)\right)$. In other words:

$$
\mathcal{I}_{d}\left(C_{f}^{\mathrm{can}}\right)=\mathcal{I}_{d}\left(\operatorname{Tor}\left(\Delta^{(1)}\right)\right) \oplus\left\langle\mathcal{F}_{d, w}\right\rangle_{w \in \mathcal{W}_{d}} .
$$

We are now ready to prove our main theorem.

TheOrem 3.2. Let $\Delta$ be a two-dimensional lattice polygon and let $f \in$ $k\left[x^{ \pm 1}, y^{ \pm 1}\right]$ be a Laurent polynomial satisfying the sufficiently generic condition from the introduction (e.g. non-degeneracy). Assume that $\Delta^{(1)}$ is two-dimensional and let $g=\sharp\left(\Delta^{(1)} \cap \mathbb{Z}^{2}\right)$.

- If $\Delta^{(2)} \neq \emptyset$ and $\Delta^{(1)} \neq \Upsilon$, then a minimal set of generators for $\mathcal{I}\left(C_{f}^{\text {can }}\right)$ is given by a basis for $\mathcal{I}_{2}\left(\operatorname{Tor}\left(\Delta^{(1)}\right)\right)$ and the quadrics $\left\{\mathcal{F}_{2, w}\right\}_{w \in \Delta^{(2)} \cap \mathbb{Z}^{2}}$.

- If $\Delta^{(1)} \cong \Upsilon$ then a minimal set of generators for $\mathcal{I}\left(C_{f}^{\mathrm{can}}\right)$ is given by the cubic defining $\operatorname{Tor}\left(\Delta^{(1)}\right) \subset \mathbb{P}^{3}$ and the quadric $\mathcal{F}_{2, w}$ with $\Delta^{(2)}=\{w\}$.

- If $\Delta^{(1)} \cong \Sigma$ then a minimal set of generators for $\mathcal{I}\left(C_{f}^{\text {can }}\right)$ is given by the single quartic $\mathcal{F}_{4, w}$ with $\left(\Delta^{(1)}\right)^{\circ} \cap\left(\frac{1}{3} \mathbb{Z}\right)^{2}=\{w\}$. 
- If $\Delta^{(1)} \cong 2 \Sigma$ then a minimal set of generators for $\mathcal{I}\left(C_{f}^{\text {can }}\right)$ is given by a basis for $\mathcal{I}_{2}\left(\operatorname{Tor}\left(\Delta^{(1)}\right)\right)$ and the three cubics $\mathcal{F}_{3, w}, \mathcal{F}_{3, w^{\prime}}, \mathcal{F}_{3, w^{\prime \prime}}$ with $\left(\Delta^{(1)}\right)^{\circ} \cap\left(\frac{1}{2} \mathbb{Z}\right)^{2}=\left\{w, w^{\prime}, w^{\prime \prime}\right\}$.

- In the other cases a minimal set of generators for the ideal $\mathcal{I}\left(C_{f}^{\text {can }}\right)$ is given by a basis for $\mathcal{I}_{2}\left(\operatorname{Tor}\left(\Delta^{(1)}\right)\right)$ and the $g-3$ cubics $\mathcal{F}_{3, w}$ with $w \in\left(\Delta^{(1)}\right)^{\circ} \cap\left(\frac{1}{2} \mathbb{Z}\right)^{2}$.

Proof. From [3, Thm. 8.1], the assumptions $\Delta^{(2)} \neq \emptyset$ and $\Delta^{(1)} \not \Upsilon$ imply that the Clifford index of $C_{f}$ is at least 2. In this case Petri's theorem [14] guarantees that $\mathcal{I}\left(C_{f}^{\text {can }}\right)$ is generated by quadrics and the statement follows from (3).

As for the other cases:

- If $\Delta^{(1)} \cong \Upsilon$, the claim follows by noting that $\operatorname{Tor}(\Upsilon)$ is cut out by the cubic $X_{-1,-1} X_{1,0} X_{0,1}-X_{0,0}^{3}$ and that a canonical curve of genus $g=4$ is of degree $2 g-2=6$, so that a single (necessarily unique) quadric suffices.

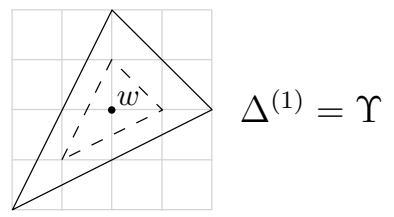

- If $\Delta^{(1)} \cong \Sigma$ or $\Delta^{(1)} \cong 2 \Sigma$ then $\operatorname{Tor}\left(\Delta^{(1)}\right) \cong \mathbb{P}^{2}$, and $C_{f}$ is a smooth plane quartic resp. a smooth plane quintic. In the quartic case the statement is obvious. As for the quintic case, by Petri's theorem we know that $\mathcal{I}\left(C_{f}^{\text {can }}\right)$ is generated by quadrics and cubics. Since $\mathcal{I}\left(\operatorname{Tor}\left(\Delta^{(1)}\right)\right)$ is generated by quadrics, the statement follows from (3). (Note that $\operatorname{Tor}\left(\Delta^{(1)}\right)$ is just the Veronese surface.)

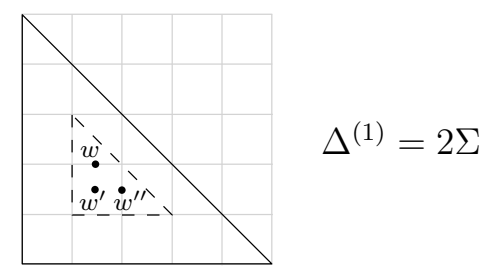

- In the other cases $C_{f}$ is a trigonal curve and $\sharp\left(\partial \Delta^{(1)} \cap \mathbb{Z}^{2}\right) \geq 4$, so that $\operatorname{Tor}\left(\Delta^{(1)}\right)$ is generated by quadrics. By Petri's theorem we know that $\mathcal{I}\left(C_{f}^{\text {can }}\right)$ is generated by quadrics and cubics, so that the statement again follows from (3). (Note that $\operatorname{Tor}\left(\Delta^{(1)}\right)$ is a rational normal surface scroll.) Remark that

$$
\sharp\left(\left(\Delta^{(1)}\right)^{\circ} \cap\left(\frac{1}{2} \mathbb{Z}\right)^{2}\right)=\sharp\left(\left(2 \Delta^{(1)}\right)^{(1)} \cap \mathbb{Z}^{2}\right)=g-3
$$


by Pick's theorem, hereby using the fact that $\Delta^{(1)}$ contains no interior lattice points.

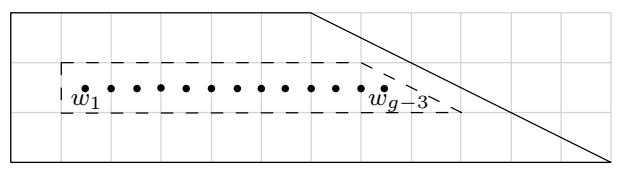

This concludes the proof.

We remark that in the last case of trigonal curves, the generators $\mathcal{F}_{3, w}$ are just the 'rolling factors' that were introduced by Reid; see [15]. For more general polygons, our forms $\mathcal{F}_{d, w}$ can be viewed as analogues of these, where the 'rolling' is done in two directions instead of one.

Theorem 3.2 immediately gives rise to an efficient algorithm for computing a minimal set of generators for the canonical ideal of $C_{f}$, for a given Laurent polynomial $f \in k\left[x^{ \pm 1}, y^{ \pm 1}\right]$ that is non-degenerate with respect to its Newton polygon $\Delta(f)$. As before we assume that

- $\sharp\left(\Delta(f)^{(1)} \cap \mathbb{Z}^{2}\right) \geq 3$, so that $C_{f}$ is of genus $g \geq 3$, and

- $\Delta(f)^{(1)}$ is two-dimensional, so that $C_{f}$ is non-hyperelliptic, or equivalently that its Clifford index is at least 1 (otherwise the canonical image is just a rational normal curve).

In case $\Delta(f)^{(1)} \cong \Sigma$ the output consists of a single quartic. If not, it consists of independent quadratic and cubic generators of the canonical ideal, i.e. $\left(\begin{array}{c}g-2 \\ 2\end{array}\right)$ quadrics and $g-3$ cubics in the case of Clifford index 1, and just $\left(\begin{array}{c}g-2 \\ 2\end{array}\right)$ quadrics in the case of Clifford index at least 2. Indeed, all one needs to do is adding the appropriate $\mathcal{F}_{d, w}$ 's to a minimal set of generators for $\operatorname{Tor}\left(\Delta^{(1)}\right)$. Finding these $\mathcal{F}_{d, w}$ 's boils down to finding relations of the form (2), which can be done by exhaustive search. An implementation can be found in the Magma file canonicalideal.m that accompanies this paper. The function of interest is called NondegIdeal().

EXAMPLE. The following sample code computes the canonical ideal of a genus 14 curve in a fraction of a second:

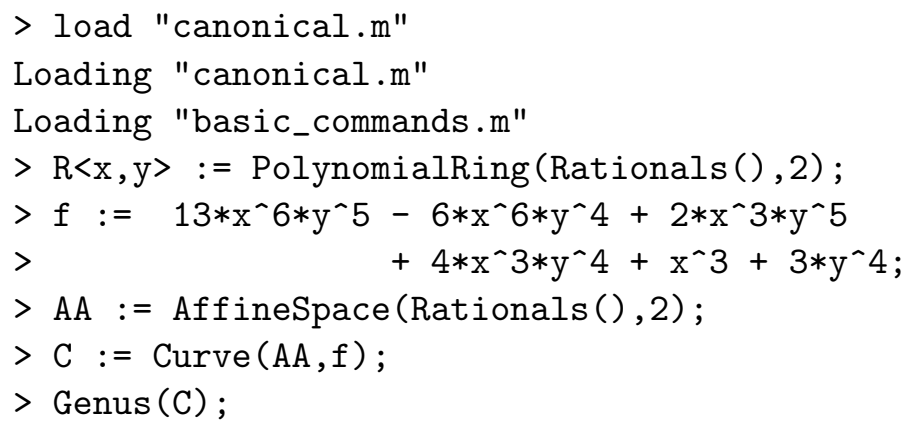


14

$>$ time I := Ideal(NondegIdeal $(f))$;

Time: 0.130

In sharp contrast, it takes the Magma intrinsic way over an hour.

$>$ time I := Ideal(Image(CanonicalMap(C)));

Time: 5405.360

Note moreover that in the latter case, in general, the output does not consist of a minimal set of generators.

Remark. Here again, the method can be slightly improved by taking into account the corresponding remark from Section 2, i.e. by computing a set of generators for $\mathcal{I}\left(\operatorname{Tor}\left(\Delta^{(1)}\right)\right)$ using Gröbner bases. It is also possible to do this at once for the entire ideal $\mathcal{I}\left(C_{f}^{\text {can }}\right.$ ), as below (continuation of the above example):

$>$ lat_points := ConvexHull(InnerPoints(NewtonPolytope (f)));

$>\mathrm{g}:=\#$ lat_points;

$>\mathrm{PP}:=\operatorname{ProjectiveSpace}(\operatorname{Rationals}(), \mathrm{g}-1)$;

$>\mathrm{phi}_{-} \mathrm{can}:=\operatorname{map}<\mathrm{C}->\mathrm{PP} \mid\left[\mathrm{x}^{\wedge} \mathrm{p}[1] * \mathrm{y}^{\wedge} \mathrm{p}[2]: \mathrm{p}\right.$ in lat_points $]>$;

$>$ time $I:=$ Ideal (Image (phi_can));

Time: 0.370

This is already much faster than the Magma intrinsic, but slower than the previous method (the difference in timing increases as the genus grows). Note again that the output does not necessarily consist of a minimal set of generators.

\section{References}

[1] Wieb Bosma, John Cannon and Catherine Playoust, 'The Magma algebra system. I. The user language', J. Symbolic Comput. (3-4) 24 (1997), 235-265 Computational algebra and number theory (London, 1993).

[2] Winfried Bruns, Joseph Gubeladze and Ngô Viêt Trung, 'Normal polytopes, triangulations, and Koszul algebras', J. Reine Angew. Math. 485 (1997), 123-160.

[3] Wouter Castryck and Filip Cools, 'Linear pencils encoded in the newton polygon' (2014) Preprint.

[4] Wouter Castryck, Jan Denef and Fréderik Vercauteren, 'Computing zeta functions of nondegenerate curves', IMRP Int. Math. Res. Pap. (2006), Art. ID 72017, 57.

[5] David Cox, John Little and Henry Schenck, Toric varieties, volume 124 of Graduate Studies in Mathematics (American Mathematical Society, Providence, RI, 2011).

[6] David Eisenbud, The geometry of syzygies, volume 229 of Graduate Texts in Mathematics, A second course in commutative algebra and algebraic geometry (SpringerVerlag, New York, 2005). 
[7] Christian Haase and Josef Schicho, 'Lattice polygons and the number $2 i+7$ ', Amer. Math. Monthly (2) 116 (2009), 151-165.

[8] Milena Hering, Syzygies of toric varieties, Thesis (Ph.D.)-University of Michigan (2006).

[9] Florian Hess, 'Computing Riemann-Roch spaces in algebraic function fields and related topics', J. Symbolic Comput. (4) 33 (2002), 425-445.

[10] Askold Hovanskiǔ, 'Newton polyhedra, and toroidal varieties', Funkcional. Anal. i Priložen. (4) 11 (1977), 56-64, 96.

[11] Robert Jan Koelman, The number of moduli of families of curves on toric surfaces, Thesis (Ph.D.)-Katholieke Universiteit Nijmegen (1991).

[12] , 'A criterion for the ideal of a projectively embedded toric surface to be generated by quadrics', Beiträge Algebra Geom. (1) 34 (1993), 57-62.

[13] - 'Generators for the ideal of a projectively embedded toric surface', Tohoku Math. J. (2) (3) 45 (1993), 385-392.

[14] Bernard Saint-Donat, 'On Petri's analysis of the linear system of quadrics through a canonical curve', Math. Ann. 206 (1973), 157-175.

[15] Jan Stevens, 'Rolling factors deformations and extensions of canonical curves', Doc. Math. 6 (2001), 185-226 (electronic).

Vakgroep Wiskunde

University of Ghent

Krijgslaan 281

9000 Gent

Belgium

wouter.castryck@gmail.com
Department of Mathematics and Applied Mathematics University of Cape Town

Private Bag X1 Rondebosch 7701

South Africa

filip.cools@uct.ac.za 